MITSUBISHI ELECTRIC RESEARCH LABORATORIES

http://www.merl.com

\title{
Efficiency and Reliability of One-Hop Broadcasting in Vehicular Ad Hoc Networks
}

\author{
Ye, F.; Yim, R.; Roy, S.; Zhang, J.
}

TR2011-013 January 2011

\begin{abstract}
In Dedicated Short Range Communications (DSRC) based vehicular networking, each vehicle periodically broadcasts control updates (that contain location and speed information etc.) to its neighbors, as a key component of traffic management and safety applications. The effectiveness of such a broadcast feature can be measured by two metrics: (1) the efficiency, or the average rate (number of nodes per sec) to which a source can deliver its broadcast packets, and (2) the reliability, or the average number of nodes that receive a specific transmission successfully. We demonstrate theoretical limits to and achievable trade-offs between efficiency and reliability for a linear network under Rayleigh fading links. We then provide power control and congestion control strategies that maximize broadcast efficiency. A strategy that achieves near-optimal broadcast efficiency when the network nodes have high mobility is also described. Ns-2 simulations are used to validate our analytical results.
\end{abstract}

IEEE Journal on Selected Areas in Communication

This work may not be copied or reproduced in whole or in part for any commercial purpose. Permission to copy in whole or in part without payment of fee is granted for nonprofit educational and research purposes provided that all such whole or partial copies include the following: a notice that such copying is by permission of Mitsubishi Electric Research Laboratories, Inc.; an acknowledgment of the authors and individual contributions to the work; and all applicable portions of the copyright notice. Copying, reproduction, or republishing for any other purpose shall require a license with payment of fee to Mitsubishi Electric Research Laboratories, Inc. All rights reserved.

Copyright (C) Mitsubishi Electric Research Laboratories, Inc., 2011

201 Broadway, Cambridge, Massachusetts 02139 



\title{
Efficiency and Reliability of One-Hop Broadcasting in Vehicular Ad Hoc Networks
}

\author{
Fei Ye, Student Member, IEEE, Raymond Yim, Member, IEEE, \\ Sumit Roy, Fellow, IEEE, Jinyun Zhang, Fellow, IEEE
}

\begin{abstract}
In Dedicated Short Range Communications (DSRC) based vehicular networking, each vehicle periodically broadcasts control updates (that contain location and speed information etc.) to its neighbors, as a key component of traffic management and safety applications. The effectiveness of such a broadcast feature can be measured by two metrics: (1) the efficiency, or the average rate (number of nodes per sec) to which a source can deliver its broadcast packets, and (2) the reliability, or the average number of nodes that receive a specific transmission successfully. We demonstrate theoretical limits to and achievable tradeoffs between efficiency and reliability for a linear network under Rayleigh fading links. We then provide power control and congestion control strategies that maximize broadcast efficiency. A strategy that achieves near-optimal broadcast efficiency when the network nodes have high mobility is also described. Ns-2 simulations are used to validate our analytical results.
\end{abstract}

Index Terms-Broadcast Efficiency, Broadcast Reliability, Congestion Control, Power Control, Rayleigh Fading, Capture Effect, CSMA, Node Density

\section{INTRODUCTION}

Improved road safety and traffic management is a fundamental driver for emerging vehicular networks [1]-[5]. As a result, the ISO Communications Architecture for Land Mobiles (CALM) [6], Society for Automotive Engineers (SAE) [7] and the IEEE Wireless Access in Vehicular Environment (WAVE) standards [8] have defined a "heart beat" message - also known as Cooperative Awareness Message (CAM) - that is required to be periodically broadcast on the control channel by all vehicles. The CAM message informs neighboring vehicles of the source locations, velocities and directions of travel, etc. and may also carry emergency alerts. These are broadcast to neighbors using Orthogonal Frequency Division Multiple (OFDM) link and Carrier Sensed Multiple Access (CSMA) MAC layer, defined in the IEEE 802.11p standard [9].

To extend transmission range and ensure successful delivery of such messages, the $802.11 \mathrm{p}$ standard allows increased transmission power on the control channel upto $33 \mathrm{dBm}$. Increasing the transmission power when only a single source transmits can improve the efficiency and reliability; however, this is not universally true in a network with multiple simultaneous broadcasts. An increase in transmit power by a source leads to corresponding increase in interference power at the receiver for other broadcasts, and leads (in dense networks) to an

F. Ye and S. Roy are with the University of Washington, Seattle, WA, USA. R. Yim and J. Zhang are with the Mitsubishi Electric Research Labs (MERL), Cambridge, MA, USA.

Emails: $\quad$ fye@u.washington.edu, yim@merl.com, sroy@u.washington.edu, jzhang@merl.com\}. interference-limited regime. Consequently, it is important to carefully study system performance which indicates how the rate of channel access by a node and the transmit power used should be adjusted according to node density, for optimal system performance.

We define two complementary performance metrics - a) the broadcast reliability, expressed as the average number of nodes that receive a specific packet transmission successfully, and b) the broadcast efficiency, or rate at which a source can deliver its broadcast packets to neighboring nodes. The VANET literature consists of several analysis of broadcast mechanisms but almost exclusively via simulation. Further, these largely concentrate on characterizing broadcast efficiency, while broadcast reliability is ignored. For example, [10] and [11] investigate broadcast efficiency for one-hop broadcast. Through simulations, it deduces a relationship between node density and per-node traffic load for maximum broadcast efficiency. Also, simulations in [12] imply that VANETs having the same communication density might have similar reception probability versus distance for a specific broadcast packet. Also, [13] and [14] derive protocols that vary transmission power so that the beaconing load in carrier sensing perceived by each vehicle does not violate a maximum beaconing load regardless of node density. [15] studies the unsaturated performance of a channel with two categories of services using distance based reception model without capture capability. Finally, mathematical expressions for broadcast performance are obtained empirically through ample simulations and least-square curve fitting in [16].

The impact of network parameters on aggregate network throughput in a single 802.11 WLAN cell has also been well studied [17]-[20]. While the performance metric in a WLAN differs from our notion of broadcast efficiency since transmissions in the former are ACK-based unicast (a single receive node per transmission), these studies shed useful light on system performance in the presence of multiple access interference, via the introduction of analytical models. For example, [18], [19] show that aggregate throughput is optimized if the contention window in 802.11 MAC protocol is scaled (inversely) with node density in a cell. In [17], the capture effect based on received signal-to-interference-plus-noise ratio (SINR) is shown to be able to increase reception probability, and hence, throughput.

In this work, we focus on developing analytical models for broadcast efficiency and reliability in 802.11 p for Rayleigh fading channels; to the the best of our knowledge, this is the first model within a VANET context to include both Rayleigh 
fading and the multi-access interference. For tractability, some simplifying assumptions were made in the analytical model; however, we validate the model results using ns- 2 simulations that consider many non-ideal effects such as hidden terminal problem. Our key results are as follows:

- The optimal broadcast efficiency and trade-off between efficiency and reliability is explicitly derived;

- The optimal power control policy is derived. For a given path loss exponent $\alpha$ and per packet transmission power $p_{0}$, the same broadcast efficiency can be achieved by scaling $p_{0}^{1 / \alpha}$ inversely with the node density (contrary to common assumptions [12], [18], [19] that broadcast efficiency is preserved by simply scaling the transmission probability inversely proportional to the node density);

- Finally, we provide the complete characterization for (1) the optimal transmission probability that maximizes broadcast efficiency when vehicle density is known, and (2) the worst-case guaranteed transmission probability when vehicle density is imprecisely known.

Since in VANET the exact node density is difficult to estimate, our ability to provide a congestion control result that guarantees a worst case performance which is very close to the optimal broadcast efficiency when the node density is exactly known, has great practical significance. Specifically, this suggests that broadcast protocols may need only gross information (e.g. whether a vehicle is in an urban or rural neighborhood) to automatically set its medium access control parameters for sharing time-critical information.

The remainder of this paper is organized as follows. The system model is introduced in Sec. II followed by the analytical formulations for broadcast efficiency and reliability over Rayleigh fading links in Sec. III. We analyze protocol behavior as a function of node density, packet length, transmission power and node transmission probability. We highlight the theoretic limits for efficiency and tradeoff between the two metrics. In Sec. IV, we provide results for the optimal broadcast efficiency when the node density is known, as well as when only loose lower and upper bounds on node density are available. In Sec. V, we suggest a congestion control algorithm that can be integrated with the IEEE 802.11 p protocol stack. Sec. VI comprises of network simulation results to demonstrate the validity of the analysis, and the paper conclude with final remarks in Sec. VII.

\section{System MODEL}

We consider a vehicular network consisting of a number of packet generating nodes (vehicles) that broadcast information to their neighbors. We assume network 'saturation' - i.e. nodes always have fixed length ( $L$ bits) packets (of same priority) waiting in their outgoing queues ${ }^{1}$. All packets are transmitted use the same transmission power of $p_{0}$ watts; all nodes access the channel with identical transmission probability $c$, and with fixed modulation and coding commensurate with transmission

\footnotetext{
${ }^{1}$ The saturation assumption is standard in network performance analysis [18] and provides a useful estimate of the maximum throughput achievable. Non-saturated queues and multi-class traffic are of interest, but beyond the scope of this paper.
}

rate over the channel of $R$ bits/sec. All vehicles are randomly positioned in a single lane, modeled by a one dimensional (1-D) homogeneous spatial Poisson point process with mean $\lambda$.

The system has a single broadcast channel that is shared amongst all nodes. The medium access behavior is modeled using $p$-persistent Carrier-Sense Multiple Access (CSMA) [21] with transmission probability $c$. In $p$-persistent CSMA, a node first senses whether the channel is free prior to channel access. If so, the node broadcasts a packet with probability $c$ regardless of the actions of the other nodes in the system. If the node chooses not to transmit when a channel is free (with probability $1-c$ ), it waits for a predefined time interval before it senses the channel again. Finally, if the channel is not free, the node reverts to receive state and attempts to decode the packet, and postpones channel sensing after this is completed.

We assume that the wireless channels undergo Rayleigh fading $^{2}$, and the path loss exponent is $\alpha$. Let $d$ be the distance between a source and a receiver, and $S$ be the received power of the transmission from a single source; then $S$ follows an exponential random variable with mean $p_{0} d^{-\alpha}$ given by the following probability distribution function (pdf):

$$
g(s)=\frac{1}{p_{0} d^{-\alpha}} \exp \left(-\frac{s}{p_{0} d^{-\alpha}}\right), \quad \forall s \geq 0 .
$$

The receiver has only single packet reception capability, and can decode the packet successfully if and only if its received SINR exceeds a threshold:

$$
P(\text { succ })=\operatorname{Pr}\left(\frac{s}{\sum_{i=1}^{\infty} I_{i}+n_{0}} \geq z\right), \quad z \geq 1,
$$

where $n_{0}$ is the noise power, $\sum_{i=1}^{\infty} I_{i}$ is the total received interference power from transmissions of neighboring nodes, and $z$ is a threshold that depends on the modulation and coding used for the packet transmission.

\section{THEORETICAL ANALYSIS}

\section{A. Broadcast Reliability}

In this work, we present two complementary views: a) a receiver centric analysis of the probability of successfully receiving a reference packet from a source whose distance from the receiver is a uniform random variable; b) a transmitter centric analysis that computes the expected number of nodes $E[N]$ that can successfully receive a reference packet from a source. Both are in presence of Rayleigh fading and multiaccess interference.

In the receiver centric analysis, we place a reference receiver at the origin and consider transmitting nodes located within $\left[-d_{m}, d_{m}\right]$ meters (see Fig. 1), where we will let $d_{m} \rightarrow \infty$ for our final result. Conditioned on the presence of a transmitter in the considered region, the source distance is uniformly distributed due to Poisson property [22]. Lemma 1 gives the distribution of the receive power due to a single transmitter without considering any interference. Then Lemma 2 computes the probability that the reference receiver successfully

\footnotetext{
${ }^{2}$ Our analysis readily adapts to other wireless channel fading models by changing the corresponding expressions for received signal power.
} 


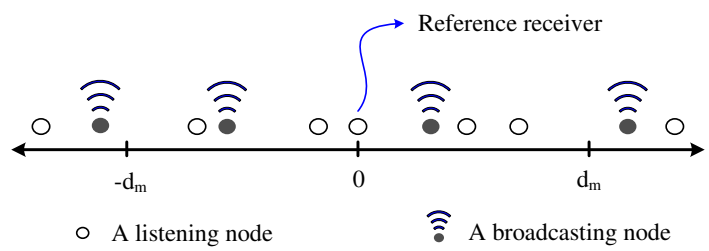

Figure 1. A receiver centric view of the 1-D broadcast network with spatially Poisson distributed nodes, for analysis in Sec.III-A.

decodes a packet from a specific source in presence of interference.

Lemma 1: The pdf $f_{S}(s)$ and cumulative distribution function (cdf) $F_{S}(s)$ of the reception power from a single transmitter that is uniformly distributed within $\left[-d_{m}, d_{m}\right]$ are given by

$$
\begin{aligned}
& f_{S}(s)=\frac{1}{d_{m} p_{0}} \int_{0}^{d_{m}} x^{\alpha} \exp \left(-p_{0}^{-1} x^{\alpha} s\right) \mathrm{d} x \\
& F_{S}(s)=1-\frac{1}{d_{m}} \int_{0}^{d_{m}} \exp \left(-p_{0}^{-1} x^{\alpha} s\right) \mathrm{d} x
\end{aligned}
$$

To compute the probability of successfully receiving a specific packet, we need to characterize the distribution of the total interference. In Appendix A, we prove that the distribution of the receive power is heavy-tailed with regularly varying tail. As a result, the convolution of the pdf's from all the interference components converges very slowly. For analytical tractability, we will approximate the total interference by the strongest interfering component, using the max-sumequivalence property [23], [24]. The following Lemma shows the probability that a receiver successfully decodes a specific packet in presence of interference.

Lemma 2: Consider a 1-D spatial network having Poisson distributed nodes with mean $\lambda$, where each node independently transmits a packet with probability $c$ in any slot. Then, the probability that a node at the origin decodes a packet successfully from a specific source node that is uniformly distributed in $\left[-d_{m}, d_{m}\right]$ is

$$
P_{\mathrm{s}-\mathrm{spec}}=\int_{z n_{0}}^{\infty} P(\operatorname{succ} \mid s) f_{S}(s) \mathrm{d} s,
$$

where $f_{S}(s)$ is given by (3), and $P(\operatorname{succ} \mid s)$ is the probability of successfully decoding a packet with receive power $s$ from the specific source in presence of interference, which is given by

$$
P(\operatorname{succ} \mid s)=\exp \left(-2 \lambda c \int_{0}^{\infty} \exp \left(-p_{0}^{-1} x^{\alpha}\left(s / z-n_{0}\right)\right) \mathrm{d} x\right)
$$

Proof: In Appendix B, Lemma 2 is proved after approximating the total interference by the strongest interfering component, using the max-sum-equivalence property.

Now, we turn to a transmitter centric view and analyze the expected number of nodes that receive a specific packet transmission successfully, denoted as the broadcast reliability.
Theorem 1: Consider a 1-D network having Poisson distributed nodes with mean $\lambda$, where each node independently transmits a packet with probability $c$ in any slot. Let $N$ be the number of nodes that decode a packet originated from the same transmission successfully, then the broadcast reliability is

$E[N]=\frac{1-c}{c z^{1 / \alpha}}\left(1-\exp \left(-2 \lambda c\left(p_{0} / n_{0}\right)^{1 / \alpha} \Gamma\left(1+\frac{1}{\alpha}\right)\right)\right)$,

where $\Gamma(x)=\int_{0}^{\infty} t^{x-1} e^{-t} \mathrm{~d} t$ is the Gamma function.

Proof: See Appendix C.

\section{B. Broadcast Efficiency}

Broadcast efficiency $U$ is the expected number of nodes to which a source delivers its packets per unit time. We will first show that the average number of nodes that a source delivers packets to at a transmission opportunity is identical to the probability that a node receives any packet successfully. When applied to reception, the broadcast efficiency is the expected number of packets that a node could receive successfully in a unit time duration. In a large network, the carrier sensing mechanism of each node reports channel status in its vicinity. While nodes in the network operate asynchronously, for the following result we assume a scenario whereby all the nodes (upon performing carrier sensing) have concluded that the channel is free at an instance.

The following corollary states the relationship between the transmitter centric and receiver centric views.

Corollary 1: The probability that a node receives and decodes a packet successfully in a slot is $c E[N]$, which equals to the expected number of nodes to which a transmitter delivers its packet successfully at a transmission opportunity.

Proof: The probability that a node receives any packet successfully can be empirically calculated by the ratio of the total number of packets successfully delivered to the total number of nodes:

$$
\begin{aligned}
& \lim _{d_{m} \rightarrow \infty} \frac{E\left[\text { Number of delivered packets in }\left[-d_{m}, d_{m}\right]\right]}{E\left[\text { Number of nodes in }\left[-d_{m}, d_{m}\right]\right]}, \\
& =\lim _{d_{m} \rightarrow \infty} \sum_{k=1}^{\infty} \frac{k\left(2 \lambda c d_{m}\right)^{k}}{k !} \cdot \frac{\exp \left(-2 \lambda c d_{m}\right) E[N]}{2 \lambda d_{m}} \\
& =c E[N] .
\end{aligned}
$$

From Theorem $1, E[N]$ is the expected number of nodes that successfully receive and decode a packet from a specific source (given that the source transmits), hence $c E[N]$ is the number of nodes on average reached by a transmitter at a transmission opportunity.

The time interval between 2 consecutive channel sensing operations at a node can be much larger than the slot duration ${ }^{3}$ defined in the 802.11 standard. For analytical convenience, we define a cycle as the (variable) time interval between 2 consecutive channel sensing operations. To evaluate broadcast efficiency, we model a node to be in one of 3 states during

\footnotetext{
${ }^{3}$ For example, in IEEE $802.11 \mathrm{p}$ networks, a slot duration is $T_{\text {slot }}=13$ microseconds.
} 


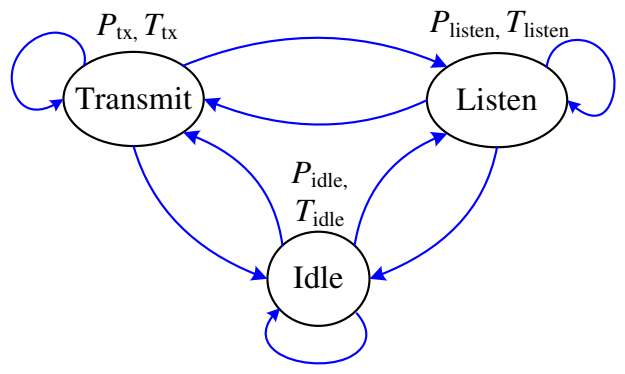

Figure 2. A node is in one of 3 states (transmit, listen, and idle) in a cycle with corresponding durations $T_{\mathrm{tx}}, T_{\text {listen, }}$, $T_{\text {idle }}$, and probabilities $P_{\mathrm{tx}}, P_{\text {listen }}, P_{\text {idle }}$.

a cycle: a) transmit, b) listen or c) idle, with corresponding durations $T_{\mathrm{tx}}, T_{\text {listen }}$, and $T_{\text {idle }}$ respectively (see Fig. 2). When a node transmits a packet, the header occupies $T_{H}$ seconds, followed by $L$ bit payload sent at channel data rate $R$ bits/second. Also, after each transmission, the node waits for a predetermined distributed inter-frame space (DIFS) time $\left(T_{\text {DIFS }}\right)$ before it senses the channel again for possible next transmission. Similarly, detects channel busy upon carrier sensing, it waits for the transmission to end ${ }^{4}$ and an additional DIFS time before it senses the channel again. Finally, when a node decides to not transmit a packet and senses that the channel is idle, it waits for a fixed time $T_{\text {slot }}$ before it makes the decision again whether to transmit or not. Hence,

$$
\begin{aligned}
T_{\mathrm{tx}} & =T_{H}+L / R+T_{\mathrm{DIFS}}, \\
T_{\text {listen }} & \approx T_{H}+L / R+T_{\mathrm{DIFS}}, \\
T_{\text {idle }} & =T_{\text {slot }} .
\end{aligned}
$$

In the physical carrier sensing model [25], a node concludes channel busy if the receive power is greater than a carrier sensing threshold $p_{\text {cs }}$. Then the effective carrier sensing range ${ }^{5}$ is calculated by $d_{\mathrm{cs}}=\left(\frac{p_{0}}{p_{\mathrm{cs}}}\right)^{1 / \alpha} \Gamma\left(1+\frac{1}{\alpha}\right)$. Due to the spatial Poisson distribution, the average node population in the physical carrier sensing range is $2 \lambda d_{\mathrm{cs}}$. Then the probability that a node transmits, receives, and is idle in a randomly chosen cycle, are respectively:

$$
\begin{aligned}
P_{\mathrm{tx}} & =c \\
P_{\text {idle }} & \approx(1-c)^{2 \lambda d_{\mathrm{cs}}}, \\
P_{\text {listen }} & =1-P_{\mathrm{tx}}-P_{\text {idle }} .
\end{aligned}
$$

The following theorem shows the broadcast efficiency.

Theorem 2: The expected number of nodes to which a source could deliver its packets in a unit time duration in a 1 -D broadcast wireless network is

$$
U=\frac{1-c}{z^{1 / \alpha}} \cdot \frac{1-\exp \left(-2 c \lambda\left(p_{0} / n_{0}\right)^{1 / \alpha} \Gamma\left(1+\frac{1}{\alpha}\right)\right)}{T_{\mathrm{tx}}-\left(T_{\mathrm{tx}}-T_{\mathrm{slot}}\right)(1-c)^{2 \lambda d_{\mathrm{cs}}}} .
$$

${ }^{4}$ Due to the hidden terminal problem, while a nearby node transmits a packet, another nearby node may transmits another packet before the end of the first transmission. Hence, in general, the listening time is longer than transmission time. However, we will omit the hidden terminal problem in the derivation.

${ }^{5}$ Due to fading, the carrier sensing range cannot be quantified by a constant value in general; however, we simplify the model and consider only the effective range.

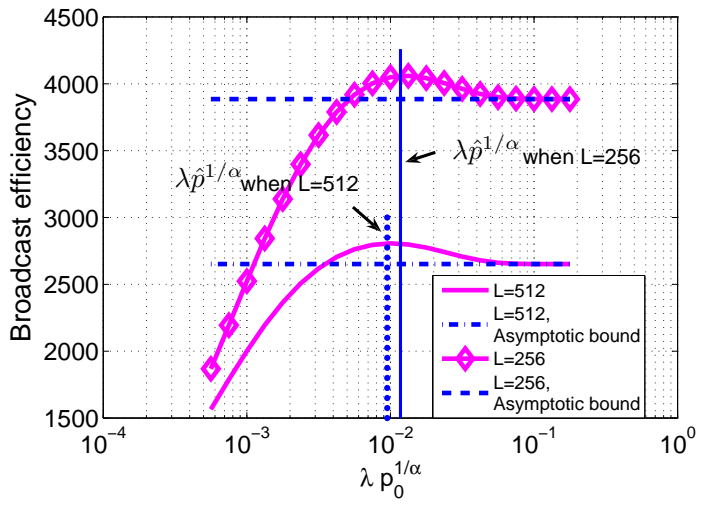

Figure 3. Broadcast efficiency as a function of $\lambda p_{0}^{1 / \alpha}$. Maximum efficiency is achieved at $\lambda \hat{p}$, where $\hat{p}$ is the optimal transmission power. $c=0.05, p_{\mathrm{Cs}}=3 n_{0}$.

Proof: By considering the expected duration of a randomly chosen cycle, we can write the broadcast efficiency as

$$
U\left(c, \lambda, p_{0}, L\right)=\frac{c E[N]}{P_{\mathrm{tx}} T_{\mathrm{tx}}+P_{\text {listen }} T_{\text {listen }}+P_{\text {idle }} T_{\text {idle }}}
$$

The result follows after substituting (9)-(11), and (12)-(13).

From Corollary 1, we see that broadcast efficiency $U$ is also the average rate in packets per second that a node receives any packets successfully. Hence, $U L$ is the average data rate in bits per second that a node successfully receives. As payload size $L$ increases, the average rate of successful reception in packets per second at a node monotonically decreases. To favor periodic heart beat message exchange in VANETs, a smaller message size is preferred.

Next, the power control policy follows from the following corollary.

Corollary 2: As a function of $\lambda p_{0}^{1 / \alpha}$, with all other parameters fixed, the broadcast efficiency in a 1-D wireless broadcast network has the following asymptotic bound:

$$
\lim _{\lambda p_{0}^{1 / \alpha} \rightarrow \infty} U=\frac{1-c}{z^{1 / \alpha}} \cdot \frac{1}{T_{\mathrm{tx}}} .
$$

When $p_{\mathrm{cs}}>n_{0}$ and for sufficiently small $c$, the optimal transmission power $\hat{p}$ is given by:

$$
\begin{aligned}
& \frac{\left(p_{\mathrm{cs}} / n_{0}\right)^{1 / \alpha}}{1-\frac{T_{\mathrm{slot}}}{T_{\mathrm{tx}}}} e^{-\lambda \eta\left(\hat{p} / n_{0}\right)^{1 / \alpha}}-e^{-\lambda \eta\left(\hat{p} / p_{\mathrm{cs}}\right)^{1 / \alpha}} \\
& =\left(\left(p_{\mathrm{cs}} / n_{0}\right)^{1 / \alpha}-1\right) e^{-\lambda \eta\left(n_{0}^{-1 / \alpha}+p_{\mathrm{cs}}^{-1 / \alpha}\right) \hat{p}^{1 / \alpha}},
\end{aligned}
$$

where $\eta=2 c \Gamma\left(1+\frac{1}{\alpha}\right)$.

Proof: Eq. (17) is immediate from (15), and (18) is obtained by considering the first order necessary condition for $(15)^{6}$.

Eq. (15) as a function of $\lambda p_{0}^{1 / \alpha}$, the asymptotic bound from (17), and the value of $\lambda \hat{p}^{1 / \alpha}$ are all plotted in Fig. 3 for two payload sizes. Theorem 2 and Corollary 2 together show an important result for power control in VANET: with all other system parameters fixed and node density $\lambda$ known,

${ }^{6}$ We use $(1-c)^{x} \approx e^{-c x}$ for small $c$, since as will be shown later in Sec. IV, the optimal transmission probability is a very small number. 


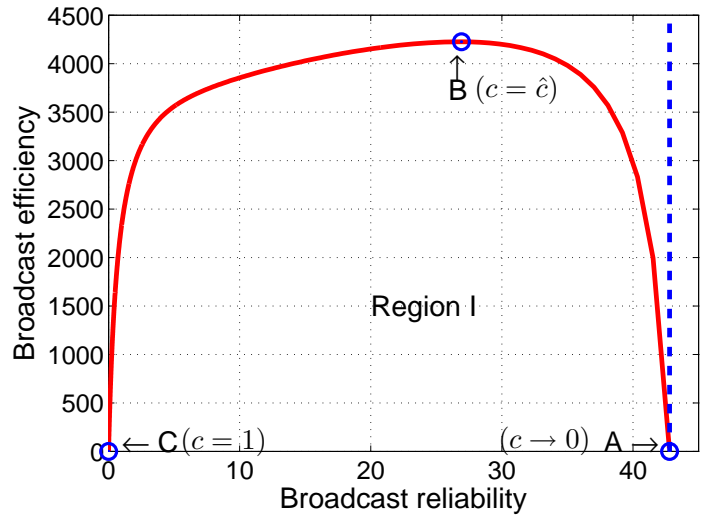

Figure 4. Tradeoff between broadcast reliability and efficiency when $\lambda=0.25, p_{0}=10^{-5} \mathrm{~W}, L=256$ bits.

nodes should set broadcast power $p_{0}=\hat{p}(\lambda)$. When node density changes, the same (optimal) broadcast efficiency can be achieved by scaling $p_{0}^{1 / \alpha}$ inversely with $\lambda$.

\section{Theoretical Limit on Tradeoff between Broadcast Reliabil- ity and Efficiency}

Thus far, we explored broadcast reliability and efficiency separately. We next capture their inter-relationship via the achievable region (Region I) in Fig. 4 that shows the tradeoff between the two factors. The maximum reliability is achieved when $c \rightarrow 0$ (point A), which corresponds to

$$
\lim _{c \rightarrow 0} E[N]=2 \lambda\left(\frac{p_{0}}{z n_{0}}\right)^{1 / \alpha} \Gamma\left(1+\frac{1}{\alpha}\right) .
$$

This is the expected number of nodes that receive a specific transmission when there is no multi-access interference. However, this comes at the expense of broadcast efficiency, as almost all nodes refrain from transmitting. As $c$ increases, the broadcast efficiency first increases due to an increase in transmission rate, but it eventually decreases again due to excessive interference. The broadcast reliability decreases monotonically as $c$ - hence the interference level - increases. Any point in region I can be achieved through time-sharing strategy. For $p$-persistent CSMA protocol, a system should operates on the line segment connecting points A and B.

\section{System OPTIMIZATION}

In the previous section, we investigated the tradeoff between broadcast reliability and efficiency, and noted that a system should operate on the line segment between points A and B in Fig. 4. In this section, we focus on point B which corresponds to the maximum broadcast efficiency, and study the optimal probability of transmission for a system with known node density, transmission power and payload size. Since VANET topology is typically dynamic, we also consider the case where the node density varies within some bounds. For such networks, we provide a worst-case guaranteed strategy for determining the probability of transmission that maintains nearoptimal broadcast efficiency over the range of node density values.

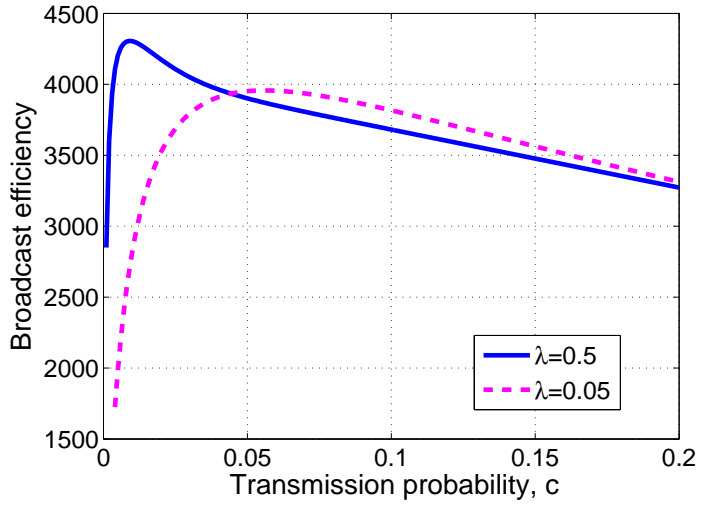

Figure 5. Broadcast efficiency versus transmission probability $c$, when $p_{0}=10^{-5} \mathrm{~W}, L=256$ bits.

\section{A. Optimal Transmission Probability}

We assume that the node density $\lambda$ is known by all nodes. In a vehicular network, such density may be estimated from traffic surveillance cameras, and the result is broadcast to all vehicles. With known $\lambda$, the optimal transmission probability $\hat{c}$ can be readily obtained by applying the first order necessary condition for stationary points of (15), which leads to the following implicit equation:

$$
\begin{aligned}
& \frac{(1-\hat{c}) 2 \lambda \xi e^{-2 \lambda \hat{c} \xi}-\left(1-e^{-2 \lambda \hat{c} \xi}\right)}{(1-\hat{c}) 2 \lambda \xi e^{-2 \lambda \hat{c} \xi}+\left(2 \lambda d_{\mathrm{cs}}-1\right)\left(1-e^{-2 \lambda \hat{c} \xi}\right)} \\
& =\left(1-\frac{T_{\text {slot }}}{T_{\mathrm{tx}}}\right)(1-\hat{c})^{2 \lambda d_{\mathrm{cs}}}
\end{aligned}
$$

where $\xi=\Gamma\left(1+\frac{1}{\alpha}\right) p_{0}^{1 / \alpha} n_{0}^{-1 / \alpha}$. Eq. (20) allows explicit computation of the optimal transmission probability $\hat{c}(\lambda)$ that each node should adopt in order to achieve maximum broadcast efficiency when node density is known. Note that the optimal transmission probability is independent of the decoding threshold $z$, but is dependent on the carrier sensing range $d_{\mathrm{cs}}$. Eq. (15) is plotted in Fig. 5 versus $c$ for different vehicle density values. As Fig. 5 shows, both the optimal transmission probability $\hat{c}$ and the absolute efficiency value achieved at $\hat{c}$ vary as density changes. We use absolute value in this figure to highlight the dependency of the maximal efficiency on vehicle density. In Fig. $6, \hat{c}(\lambda)$ derived from (20) is plotted as a function of vehicle density. The optimal transmission probability $\hat{c}(\lambda)$ is monotonically decreasing, implying smaller transmission probability for networks of higher density. However, the optimal transmission probability does not scale inversely proportional to the node density.

\section{B. Worst-Case Guaranteed Transmission Probability}

We now assume that it is impossible to estimate and distribute the node density accurately to the nodes in the network. Rather, only loose lower and upper bounds of the density $0 \leq \lambda_{1} \leq \lambda \leq \lambda_{2}$ are known. We seek a transmission probability that performs as close to the optimal broadcast efficiency as possible for any actual node density within the 


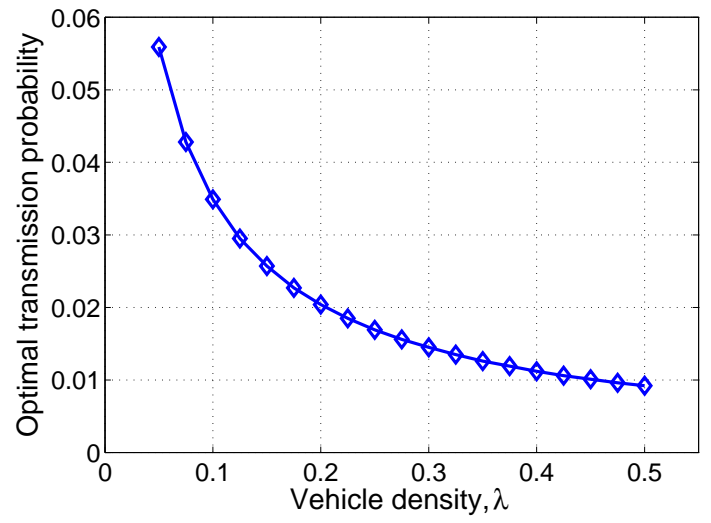

Figure 6. Optimal transmission probability $\hat{c}(\lambda)$ as a function of $\lambda$, when $p_{0}=10^{-5} \mathrm{~W}, L=256$ bits.

bounds. Hence,

$$
\hat{c}\left(\lambda_{1}, \lambda_{2}\right)=\arg \max _{c} \min \left\{\frac{U(c, \lambda)}{U(\hat{c}(\lambda), \lambda)}: \forall \lambda \in\left[\lambda_{1}, \lambda_{2}\right]\right\} .
$$

where $0 \leq \frac{U(c, \lambda)}{U(\hat{c}(\lambda), \lambda)} \leq 1$ is the normalized broadcast efficiency, which measures the efficiency with respect to what is optimally achievable. Note that the optimal transmission probability at exact node density $\lambda$ is $\hat{c}(\lambda)=\hat{c}(\lambda, \lambda)$.

From the optimal result (Fig. 6), we know that the optimal transmission probability decreases as a function of $\lambda$, and it can be shown that the broadcast efficiency decreases monotonically as the transmission probability deviates from the optimal value for a given node density. Hence, the worst-case guaranteed transmission probability can be obtained by simply considering the intersection of $\frac{U\left(c, \lambda_{1}\right)}{U\left(\hat{c}\left(\lambda_{1}\right), \lambda_{1}\right)}$ and $\frac{U\left(c, \lambda_{2}\right)}{U\left(\hat{c}\left(\lambda_{2}\right), \lambda_{2}\right)}$. This leads to the following implicit equation:

$$
\begin{aligned}
& \frac{\left(1-e^{-2 \lambda_{2} \xi \hat{c}\left(\lambda_{1}, \lambda_{2}\right)}\right)\left(1-\left(1-\frac{T_{\text {slot }}}{T_{\mathrm{tx}}}\right)\left(1-\hat{c}\left(\lambda_{1}, \lambda_{2}\right)\right)^{2 \lambda_{1} d_{c s}}\right)}{\left(1-e^{-2 \lambda_{1} \xi \hat{c}\left(\lambda_{1}, \lambda_{2}\right)}\right)\left(1-\left(1-\frac{T_{\text {slot }}}{T_{\mathrm{tx}}}\right)\left(1-\hat{c}\left(\lambda_{1}, \lambda_{2}\right)\right)^{2 \lambda_{2} d_{c s}}\right)} \\
& =\frac{U\left(\hat{c}\left(\lambda_{2}\right), \lambda_{2}\right)}{U\left(\hat{c}\left(\lambda_{1}\right), \lambda_{1}\right)} .
\end{aligned}
$$

The equipotential surface of the normalized worst-case guaranteed broadcast efficiency is plotted in Fig. 7 for a wide range of $\lambda_{1}$ and $\lambda_{2}$. Even when the lower and upper bounds of the node density differ by an order of magnitude, the worstcase guaranteed solution is at least $95 \%$ of what is optimally achievable under the current parameter settings. Note that while the broadcast efficiency remains high, the worst-case guaranteed solution has an impact on broadcast reliability. Specifically, if the true density is closer to the upper bound, the worst-case guaranteed solution would be more aggressive than the optimal. As a results, each transmission reaches less number of neighboring nodes.

\section{Congestion Control}

Thus far, we have established the worst-case guaranteed transmission probability, and in the limit case the optimal transmission probability, given a range of possible node density values. The transmission probability for 802.11 MAC

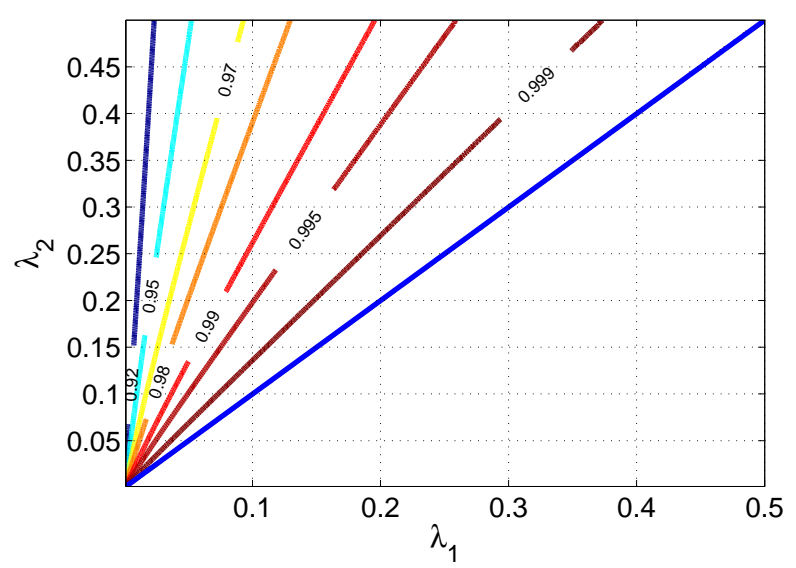

Figure 7. Contour lines of the normalized worst-case broadcast efficiency on the $\lambda_{1}-\lambda_{2}$ plane.

protocol [26] is known to be related to the (fixed and/or variable) size of the contention window that is used to regulate channel access by nodes [18] [19]. For example, a packet at the head of the MAC layer queue uniformly chooses a backoff timer value between $[0, W-1]$, and the packet is only transmitted after the node senses a sufficient amount of channel inactivity. Typically, for unicast where data link control is based on receipt of acknowledgements, the contention window size is increased in response to loss of any acknowledgement. However, for broadcast mode in 802.11, there does not exist any feedback mechanism for nodes to detect a collision. In fact, the contention window size is a predetermined fixed value according to the packet class in 802.11 EDCA or $802.11 \mathrm{p}$ for broadcast packets. More relevantly for the VANET context, the contention window size does not change according to node density $\lambda$, transmission power $p_{0}$, and payload size $L$. Hence, as the node density changes in a dynamic network, the broadcast efficiency may suffer drastically if the contention window size is set incorrectly.

In this section, we offer congestion control mechanisms that may be used to regulate the channel access probability for a node. First, we propose directly changing the contention window size; however this would require changes to the 802.11p standard and is not generally recommended. Alternatively, as per Fig. 8, we propose to insert the Congestion Control Layer above the short message protocol standard (such as WAVE Short Message Protocol (WSMP) layer in the WAVE communication protocol stack [8]). The new congestion control layer is similar to the transmission control protocol (TCP) layer for Internet Protocol (IP) traffic. But unlike TCP, it is not connection oriented; rather, it regulates broadcast traffic on the control channel without observing any acknowledgements. In one solution, the congestion control layer receives signalling information from the MAC layer, and uses that to regulate access probability of outgoing traffic. Alternately, the congestion control algorithm may regulate the outgoing traffic without any input from the MAC layer.

\section{A. Direct MAC Layer Congestion Control}

From [18] [19], $c=\frac{2}{W+1}$ is the relationship between the outgoing packet probability $c$ and the fixed contention window 
size $W$ for broadcast packets. Let $\hat{c}\left(\lambda_{1}, \lambda_{2}\right)$ be the worst-case guaranteed transmission probability, then the corresponding worst-case guaranteed contention window size $\hat{W}\left(\lambda_{1}, \lambda_{2}\right)$ is given by

$$
\hat{W}\left(\lambda_{1}, \lambda_{2}\right)=\left\lceil\frac{2}{\hat{c}\left(\lambda_{1}, \lambda_{2}\right)}-1\right\rceil,
$$

where $\lceil\cdot\rceil$ is the ceiling function.

\section{B. Congestion Control Layer with MAC Signaling}

Direct manipulation of the contention window size in MAC layer is sometimes not feasible. Instead, we design a congestion control layer, a practical solution which minimizes the interaction with MAC layer to retain MAC's integrity. Fig. 8 shows the congestion control layer in relations to the other elements in a WAVE communication protocol stack. We see that the congestion control layer is analogous to TCP, except that it regulates broadcast traffic that is sent to the short message layer. An additional queue is implemented in the congestion control layer in addition to the MAC layer queue, and a control interface between the congestion control layer and MAC layer is optional.

The desired transmission probability $\hat{c}\left(\lambda_{1}, \lambda_{2}\right)$ at every transmission opportunity is derived in (21). However, the transmission opportunity occurs at irregular time interval, depending on whether the node is transmitting a packet, and whether there are any ongoing nearby transmissions. With MAC signaling, the congestion control layer learns when the MAC layer senses a channel idle, and when a packet is transmitted. It stores all packets in its queue, and sends a packet to the short message layer with probability $q$ at every transmission opportunity. To achieve the effective transmission probability $\hat{c}\left(\lambda_{1}, \lambda_{2}\right)$, the congestion control layer should set

$$
q= \begin{cases}\frac{2 \hat{c}\left(\lambda_{1}, \lambda_{2}\right)}{2-\hat{c}\left(\lambda_{1}, \lambda_{2}\right)(W-1)}, & \hat{c}\left(\lambda_{1}, \lambda_{2}\right) \in\left[0, \frac{2}{W+1}\right), \\ 1, & \hat{c}\left(\lambda_{1}, \lambda_{2}\right) \in\left[\frac{2}{W+1}, 1\right] .\end{cases}
$$

This collaborative transmission control operation is summarized below in Alg. 1 .

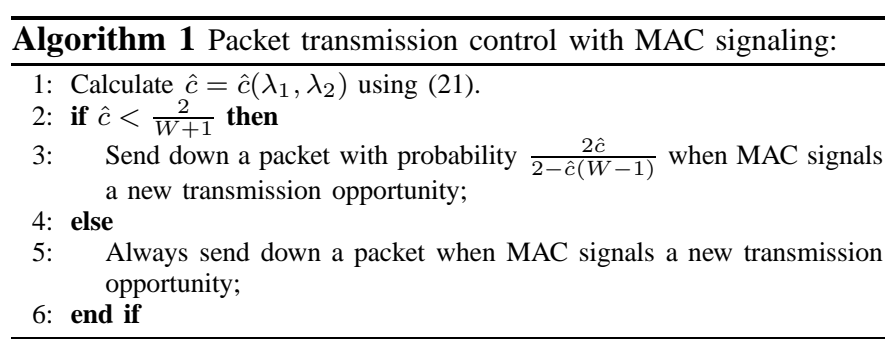

\section{Congestion Control Layer without MAC Signaling}

Without MAC signaling, congestion control effectively becomes a rate control problem. The congestion control layer may simply controls the rate at which packets are sent to the short message layer using vehicle density information. Specifically, the desired transmission data rate in packets per second is a function of node density $\lambda$ :

$$
\rho(\lambda)=\frac{\hat{c}(\lambda)}{T_{\mathrm{tx}}-\left(T_{\mathrm{tx}}-T_{\mathrm{slot}}\right)(1-\hat{c}(\lambda))^{2 \lambda d_{\mathrm{cs}}}} .
$$

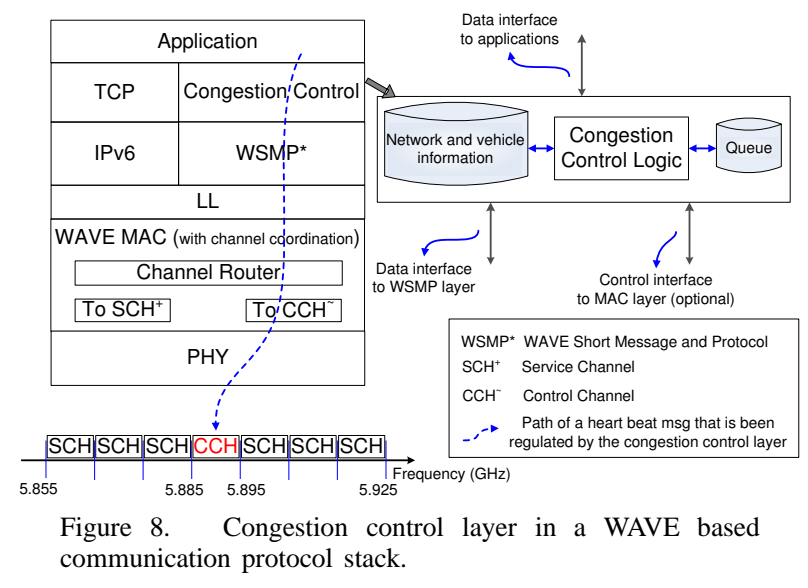

\section{Simulation}

We simulate a 1-D vehicular network using parameters (Table I) from WAVE/802.11p standards. Various vehicle densities and contention window sizes are evaluated. The simulation is conducted using ns-2 [27], with improved wireless channel, physical layer and MAC layer abstractions from the patch provided by [28]. The underlying physical layer monitors the accumulated interference level and adopts a SINR based capture model for packet reception. The contention window operations closely follow the IEEE $802.11 \mathrm{p}$ standard. This simulator has been widely used in VANET simulations [10][12], [29]. Furthermore, the hidden terminal effect exists in all simulations.

Fig. 9 plots the simulated normalized broadcast efficiency $\frac{U}{U(\hat{c}(\lambda))}$ and (15), when accurate vehicle density is known. As we can see, the optimal contention window size matches well with the simulation result. Without normalization to the maximum value, we find that the simulated broadcast efficiency is generally less than the theoretical value. This is because the theoretical analysis approximates the total interference by the strongest interfering component and assumes synchronicity of transmission opportunities, both are optimistic assumptions that over-estimates the efficiency. Nonetheless, the theoretical result accurately predicts the optimal contention window size, which is valuable for system designers.

In Fig. 10, we consider a wide range of vehicle densities $\lambda \in\{0.05,0.25,0.5\}$ (veh/m), which corresponds to mean

Table I

SIMULATION PARAMETERS

\begin{tabular}{l|l} 
Parameter & Value \\
\hline \hline Central frequency & $5.890 \mathrm{Ghz}$ \\
Channel bandwidth & $10 \mathrm{Mhz}$ \\
Channel data rate & $3 \mathrm{Mbps}$ \\
Channel model & Rayleigh fading \\
Path loss & 4 \\
Modulation scheme & BPSK \\
Capture threshold for BPSK & $5 \mathrm{~dB}$ \\
Slot time & $13 \mathrm{us}$ \\
Header duration & $40 \mathrm{us}$ \\
Symbol duration & $8 \mathrm{us}$ \\
SIFS & $32 \mathrm{us}$ \\
Data packet size & $51 \mathrm{Bytes}$ \\
Transmission power & $1 \mathrm{e}-5 \mathrm{Watt}$ \\
\hline
\end{tabular}




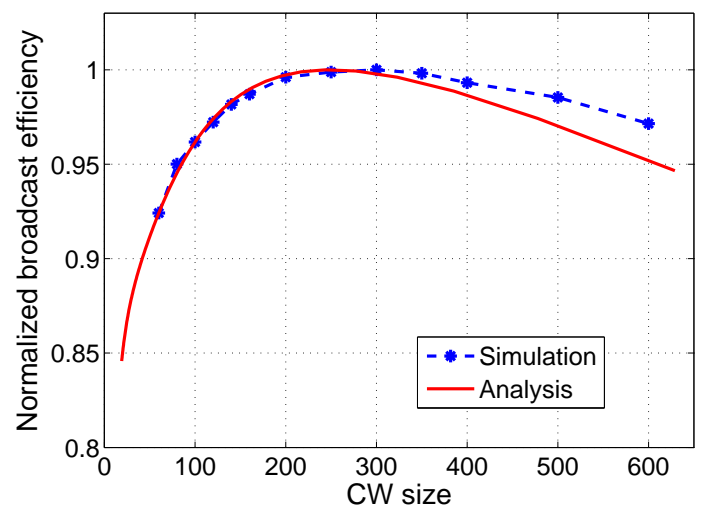

Figure 9. Normalized broadcast efficiency from analysis and simulation when density $\lambda=0.5$.

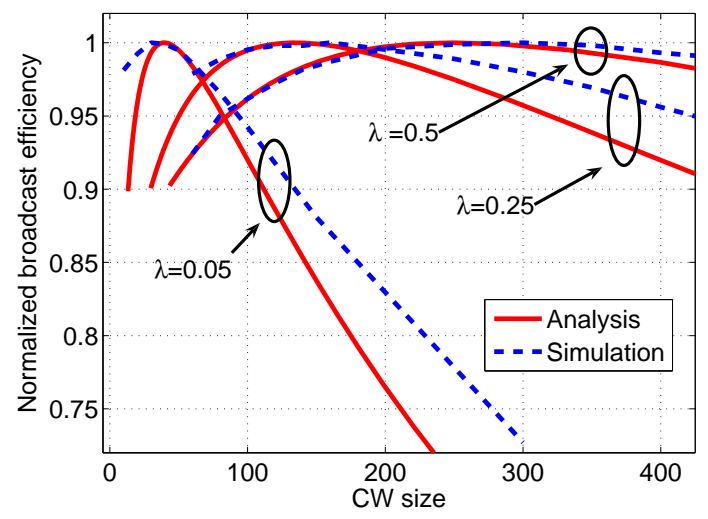

Figure 10. Normalized broadcast efficiency for a wide range of densities from analysis and simulation. inter-vehicle spacing of 8,40 and $80(\mathrm{~m})$ on a 4-lane freeway. The maximum (absolute) broadcast efficiency, as a function of vehicle density, is unknown to individual vehicles due to inaccurate density knowledge. However, for worst-case guaranteed performance, the system sets the contention window size $W$ so to maximize the normalized broadcast efficiency over a range of possible vehicle densities. For the given parameters, the worst-case guaranteed solution for $\lambda \in\{0.05,0.5\}$ should set $W=85$, which is the cross-over point of the curves for $\lambda=0.05$ and $\lambda=0.5$. At $W=85$, if the true density is $\lambda=0.05$, we see from the simulation result that, while the chosen contention window is larger than optimal value, the broadcast efficiency is still $96 \%$ of the optimal efficiency that is achievable for systems with $\lambda=0.05$. On the other hand, if the true density is $\lambda=0.5$, while $W=85$ is lower than the would-be optimal value, the system is achieving $95 \%$ of the optimal broadcast efficiency. If the system can further decides between $\lambda \in[0.25,0.5]$ (urban scenario) and $\lambda \in[0.05,0.25]$ (rural scenario), then $97 \%$ and $99 \%$ of the optimal performance can be guaranteed, respectively.

From Fig. 9 and 10, we observe a mismatch of simulated and analytical efficiency results when contention window size is far from the optimal; this effect is particularly pronounced when the vehicle density is small. Part of this discrepancy is a simulation artifact for low vehicle density, as it requires a longer simulation interval than we have used.

\section{CONCLUSION}

In this paper, we introduced broadcast reliability that measures the average number of nodes that receive a specific packet transmission successfully, and broadcast efficiency that measures the average rate at which nodes receive any broadcast packets successfully. We analyzed behavior in a Rayleigh fading channel, and validated its accuracy using ns-2 simulations. The analysis provides the following fundamental results: (1) an achievable region and consequent tradeoff between broadcast reliability and efficiency; (2) a power control policy as a function of node density; (3) complete characterization of the optimal transmission probability as a function of node density, transmission power, packet length and other VANET system parameters; and (4) a worst-case guaranteed transmission strategy for nodes with high mobility that shows near-optimal broadcast efficiency ( $95 \%$ of optimal efficiency in simulation) can be achieved.

\section{APPENDIX A}

\section{PROOF OF THE RECEIVE POWER'S HEAVY-TAIL PROPERTY}

A distribution is said to have a regularly varying tail with index $\gamma \geq 0$ if and only if its cdf $F(x)$ satisfies $\lim _{x \rightarrow \infty} \frac{1-F(x y)}{1-F(x)}=y^{-\gamma}$ for any $y>0$. From (4), we have the cdf $F_{S}(s)$ of the receive power $S$ from a single transmitter that is uniformly distributed in $\left[-d_{m}, d_{m}\right]$. We first change the variable in (4) using $t=p_{0}^{-1} x^{\alpha} s$,

$$
F_{S}(s)=1-d_{m}^{-1} \alpha^{-1} p_{0}^{1 / \alpha} s^{-1 / \alpha} \int_{0}^{p_{0}^{-1} s d_{m}^{\alpha}} t^{-1+1 / \alpha} e^{-t} \mathrm{~d} t .
$$

Then we apply (26) to the heavy tail definition,

$$
\begin{aligned}
\lim _{x \rightarrow \infty} \frac{1-F_{S}(x y)}{1-F_{S}(x)} & =\lim _{x \rightarrow \infty} \frac{(x y)^{-1 / \alpha}}{x^{-1 / \alpha}} \cdot \frac{\int_{0}^{p_{0}^{-1} x y d_{m}^{\alpha}} t^{-1+1 / \alpha} e^{-t} \mathrm{~d} t}{\int_{0}^{p_{0}^{-1} x d_{m}^{\alpha}} t^{-1+1 / \alpha} e^{-t} \mathrm{~d} t} \\
& =y^{-1 / \alpha}
\end{aligned}
$$

Hence, we have proved that the distribution of the receive power is heavy-tailed, and has a regularly varying tail with index $\frac{1}{\alpha}$.

\section{APPENDIX B \\ PROOF OF LEMMA 2}

In the receiver centric view, we first consider the distribution of the interference power heard by the reference receiver at the origin. From (3) and (4), it can be shown that the distribution of the receive power from a single transmitter is heavy-tailed with regularly varying tail. Hence, for analytical tractability, we will approximate the total interference by the strongest interfering component, using the max-sum-equivalence property [23], [24]. Let $K_{1}$ be the number of transmitting nodes in $\left[-d_{m}, d_{m}\right]$, then $K_{1}$ is a poisson random variable with mean $2 \lambda c d_{m}$ due to Poisson's splitting property. For a given value $k_{1}$ of the random variable $K_{1}$, the cdf of maximum interference is thus given by the largest order statistic of $F_{S}(s)$ over a population size of $k_{1}$, which equals to $\left(F_{S}(s)\right)^{k_{1}}$. 
Now, consider a specific packet that is received with signal power $s \geq z n_{0}$, we compute the conditional probability of receiving this packet successfully based on received SINR,

$$
\begin{aligned}
P(\operatorname{succ} \mid s) & \\
= & \lim _{d_{m} \rightarrow \infty} \sum_{k_{1}=0}^{\infty} \frac{\left(2 \lambda c d_{m}\right)^{k_{1}}}{k_{1} !} e^{-2 \lambda c d_{m}} \operatorname{Pr}\left(\sum_{i=1}^{k_{1}} I_{i} \leq z^{-1} s-n_{0}\right), \\
\approx & \lim _{d_{m} \rightarrow \infty} \sum_{k_{1}=0}^{\infty} \frac{\left(2 \lambda c d_{m}\right)^{k_{1}}}{k_{1} !} e^{-2 \lambda c d_{m}} \\
& \cdot \operatorname{Pr}\left(\max \left\{I_{1}, I_{2}, \cdots, I_{k_{1}}\right\} \leq z^{-1} s-n_{0}\right), \\
= & \lim _{d_{m} \rightarrow \infty} \sum_{k_{1}=0}^{\infty} \frac{\left(2 \lambda c d_{m}\right)^{k}}{k_{1} !} e^{-2 \lambda c d_{m}}\left(F_{S}\left(z^{-1} s-n_{0}\right)\right)^{k_{1}} \\
= & \lim _{d_{m} \rightarrow \infty} \exp \left(-2 \lambda c d_{m}\left(1-F_{S}\left(z^{-1} s-n_{0}\right)\right)\right) \\
= & \exp \left(-2 \lambda c \int_{0}^{\infty} \exp \left(-p_{0}^{-1} x^{\alpha}\left(z^{-1} s-n_{0}\right)\right) \mathrm{d} x\right) .
\end{aligned}
$$

In step 2, the approximation follows from the max-sumequivalence property of heavy-tailed distribution. Finally, eq. (5) follows from Lemma 1, noting that the pdf of the receive power of the specific packet from the source is simply $f_{S}(s)$, and a successful packet reception requires a minimum received signal power of $z n_{0}$.

\section{APPENDIX C \\ PROOF OF THEOREM 1}

In the transmitter centric view, we place a reference transmitting node at the original, and consider the region $\left[-d_{m}, d_{m}\right]$. Conditioned on a transmission by the reference node, a node in the considered region has probability $1-c$ of listening to this transmission. Due to Poisson's splitting property, the number of listening nodes in the considered region $K_{2}$, is a Poisson random variable with mean $2 \lambda(1-c) d_{m}$. Condition on the knowledge of $K_{2}$, these listening nodes are uniformly distributed in the considered region. Hence, $P_{\text {s-spec }}$ in Lemma 2 shows the probability of that a listening node receives the packet successfully from the reference node. Hence, the expected number of nodes that can successfully receive and decode a specific packet from the reference node is derived in (29). In the last step, we use approximation $z^{-1} s-n_{0} \approx z^{-1} s$. The result follows after performing integration by parts and simplification.

\section{REFERENCES}

[1] F. Li and Y. Wang, "Routing in vehicular ad hoc networks: a survey," IEEE Veh. Technol. Mag., vol. 2, no. 2, pp. 12-22, June 2007.

[2] N. Wisitpongphan, F. Bai, P. Mudalige, V. Sadekar, and O. Tonguz, "Routing in sparse vehicular ad hoc wireless networks," IEEE Journal on Selected Areas in Communications, vol. 25, no. 8, pp. 1538-1556, 2007.

[3] J. Zhu and S. Roy, "MAC for dedicated short range communications in intelligent transport system," IEEE Communication Magazine, vol. 41, no. 12, pp. 60-67, 2003.

[4] R. Yim, J. Guo, P. Orlik, and J. Zhang, "Received power-based prioritized rebroadcasting for V2V safety message dissemination," in Proc. of Int. Transport. Sys. World Congr., Sept. 2009.
[5] J. Yin, T. ElBatt, G. Yeung, B. Ryu, S. Habermas, H. Krishnan, and T. Talty, "Performance evaluation of safety applications over DSRC vehicular ad hoc networks," in Proc. of the 1st ACM VANET workshop, 2004, pp. 1-9.

[6] "ISO Std, Communications, Air-interface, Long and Medium rang (CALM)," http://www.isotc204wg16.org/.

[7] "ITS Std SAE J2735 - Dedicated Short Range Communications (DSRC) Message Set Dictionary," http://www.standards.its.dot.gov/.

[8] "IEEE Std 1609 family, IEEE Trial-Use Standard for Wireless Access in Vehicular Environments(WAVE)," Nov. 2006.

[9] "IEEE P802.11p/D5.0, IEEE 802.11 Amendment 7: Wireless Access in Vehicular Environments," Nov. 2008.

[10] M. Torrent-Moreno, D. Jiang, and H. Hartenstein, "Broadcast reception rates and effects of priority access in 802.11-based vehicular ad-hoc networks," in Proc. of the 1st ACM VANET workshop, 2004, pp. 10-18.

[11] M. Torrent-Moreno, S. Corroy, F. Schmidt-Eisenlohr, and H. Hartenstein, "IEEE 802.11-based one-hop broadcast communications: understanding transmission success and failure under different radio propagation environments," in Proc. of the 9th ACM MSWiM, 2006, pp. 68-77.

[12] D. Jiang, Q. Chen, and L. Delgrossi, "Communication density: a channel load metric for vehicular communications research," in Proc. of IEEE Int conf. on Mobile Adhoc and Sensor Systems (MASS), 2007, pp. 1-8.

[13] J. Mittag, F. Schmidt-Eisenlohr, M. Killat, J. Härri, and H. Hartenstein, "Analysis and design of effective and low-overhead transmission power control for VANETs," in Proc. of the 5th ACM VANET workshop, 2008, pp. 39-48.

[14] M. Torrent-Moreno, P. Santi, and H. Hartenstein, "Fair sharing of bandwidth in VANETs," in Proc. of the 2nd ACM VANET workshop, 2005, pp. 49-58.

[15] X. Ma, X. Chen, and H. Refai, "Performance and reliability of DSRC vehicular safety communication: a formal analysis," EURASIP Journal on Wireless Communications and Networking, pp. 1-13, 2009.

[16] M. Killat and H. Hartenstein, "An empirical model for probability of packet reception in vehicular ad hoc networks," EURASIP Journal on Wireless Communications and Networking, 2009.

[17] M. Zorzi and R. Rao, "Capture and retransmission control in mobile radio," IEEE Journal on Selected Areas in Communications, vol. 12, pp. 1289-1298, 1994

[18] G. Bianchi, "Performance analysis of the IEEE 802.11 distributed coordination function," IEEE Journal on Selected Areas in Communications, vol. 18 , no. 3, pp. 535-547, 2000.

[19] F. Calì, M. Conti, and E. Gregori, "Dynamic tuning of the IEEE 802.11 protocol to achieve a theoretical throughput limit," IEEE/ACM Trans. on Networking, vol. 8, no. 6, pp. 785-799, 2000.

[20] J. Zhu, B. Metzler, X. Guo, and Y. Liu, "Adaptive CSMA for scalable network capacity in high-density WLAN: a hardware prototyping approach," in Proc. of INFOCOM, 2006, pp. 1-10.

[21] L. Kleinrock and F. Tobagi, "Packet switching in radio channels: Part I-carrier sense multiple-access modes and their throughput-delay characteristics," IEEE Trans. on Communications, vol. 23, no. 12, pp. 1400-1416, 1975.

[22] S. Ross, Introduction to probability models. Academic Press, 2007.

[23] J. Cai and Q. Tang, "On max-sum equivalence and convolution closure of heavy-tailed distributions and their applications," Journal of Applied Probability, vol. 41, no. 1, pp. 117-130, 2004.

[24] S. Asmussen, Ruin probabilities. World Scientific Singapore, 2000.

[25] J. Zhu, X. Guo, L. L. Yang, W. Steven Conner, S. Roy, and M. Hazra, "Adapting physical carrier sensing to maximize spatial reuse in 802.11 mesh networks," Wireless Communications and Mobile Computing, vol. 4, no. 8, pp. 933-946, 2004.

[26] "IEEE Std 802.11-2007, Wireless LAN Medium Access Control (MAC) and Physical Layer (PHY) Specifications," 2007.

[27] "ns-2: the network simulator version 2," http://www.isi.edu/nsnam/ns.

[28] Q. Chen, F. Schmidt-Eisenlohr, D. Jiang, M. Torrent-Moreno, L. Delgrossi, and H. Hartenstein, "Overhaul of IEEE 802.11 modeling and simulation in ns-2," in Proc. of the 10th ACM MSWiM.

[29] D. Jiang, Q. Chen, and L. Delgrossi, "Optimal data rate selection for vehicle safety communications," in Proc. of the 5th ACM VANET workshop, 2008, pp. 30-38. 


$$
\begin{aligned}
E[N] & =\lim _{d_{m} \rightarrow \infty} E_{K_{2}}\left[K_{2} P_{\mathrm{s} \text {-spec }}\right] \\
& =\lim _{d_{m} \rightarrow \infty} 2 \lambda(1-c) d_{m} P_{\mathrm{s}-\mathrm{spec}} \\
& =2 \lambda(1-c) \int_{z n_{0}}^{\infty}\left(\int_{0}^{\infty} p_{0}^{-1} d^{\alpha} \exp \left(-p_{0}^{-1} d^{\alpha} s\right) \mathrm{d} d\right) \exp \left(-2 \lambda c \int_{0}^{\infty} \exp \left(-p_{0}^{-1} d^{\alpha}\left(z^{-1} s-n_{0}\right)\right) \mathrm{d} d\right) \mathrm{d} s \\
& =2 \lambda(1-c) \int_{z n_{0}}^{\infty} p_{0}^{1 / \alpha} \frac{1}{\alpha} \Gamma\left(1+\frac{1}{\alpha}\right) s^{-1-1 / \alpha} \exp \left(-2 \lambda c\left(z^{-1} s-n_{0}\right)^{-1 / \alpha} p_{0}^{1 / \alpha} \frac{1}{\alpha} \Gamma\left(\frac{1}{\alpha}\right)\right) \mathrm{d} s \\
& \approx 2 \lambda(1-c) \int_{z n_{0}}^{\infty} p_{0}^{1 / \alpha} \frac{1}{\alpha} \Gamma\left(1+\frac{1}{\alpha}\right) s^{-1-1 / \alpha} \exp \left(-2 \lambda c z^{1 / \alpha} p_{0}^{1 / \alpha} \Gamma\left(1+\frac{1}{\alpha}\right) s^{-1 / \alpha}\right) \mathrm{d} s
\end{aligned}
$$

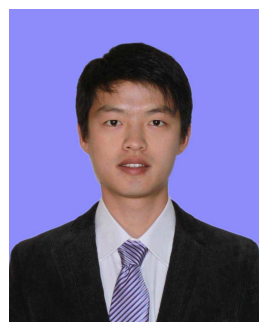

Fei Ye received the B.S. and M.Sc. degrees, both in electronic engineering, from Tsinghua University, Beijing, China, in 2005 and 2007, respectively. Since September 2007, he has been working towards the $\mathrm{Ph} . \mathrm{D}$. degree in electrical engineering at the University of Washington, Seattle.

His main research interest is protocol design in wireless networks including wireless LAN, wireless mesh, vehicular network, focusing on performance optimization at different layers, i.e., network layer (e.g., multi-hop routing), and MAC layer (e.g., IEEE802.11p). His current work is on MAC design and packet level error correction for safety application and data dissemination in vehicular networks.

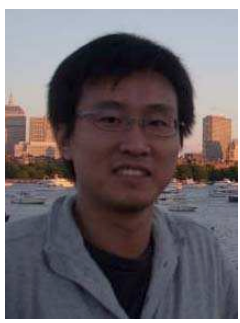

Raymond Yim (S'0-M'7) received his B. Eng. and M. Eng. degrees in electrical and computer engineering from McGill University in Canada, and Ph.D. from Harvard University. In 2006-2007, he was an assistant professor in electrical and computer engineering at F. W. Olin College of Eng., Needham, MA. Since 2008, he has been a research scientist at the Mitsubishi Electric Research Laboratories, Cambridge, MA. His research interests include the design and analysis of cross-layered architectures and protocols for wireless communication networks, multiple access protocols, link adaptation, cooperative communications, smart antenna systems, interference management and intelligent transportation systems.

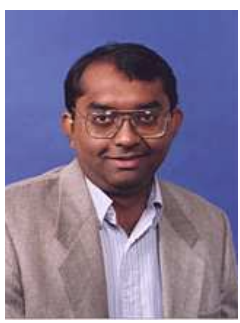

Sumit Roy received the B. Tech. degree from the Indian Institute of Technology (Kanpur) in 1983, and the M. S. and Ph. D. degrees from the University of California (Santa Barbara), all in Electrical Engineering in 1985 and 1988 respectively, as well as an M. A. in Statistics and Applied Probability in 1988. Presently he is Professor and Assoc. Chair of Electrical Engineering, Univ. of Washington where his research interests include analysis/design of wireless communication and sensor network systems with a diverse emphasis on various technologies: wireless LANs (802.11) and wireless MANs (802.16), multi-standard wireless internetworking and cognitive radio platforms, vehicular and underwater networks, and sensor networking involving RFID technology.

He spent 2001-03 on academic leave at Intel Wireless Technology Lab as a Senior Researcher engaged in systems architecture and standards development for ultra-wideband systems (Wireless PANs) and next generation high-speed wireless LANs. During Jan-July 2008, he was Science Foundation of Ireland's E.T.S. Walton Awardee for a sabbatical at University College, Dublin. His activities for the IEEE Communications Society (ComSoc) includes membership of several technical and conference program committees, notably the Technical Committee on Cognitive Networks. He currently serves on the Editorial Board for IEEE Trans. Communications, IEEE Intelligent Transportation Systems and IEEE Trans. Smart Grids. He was elevated to IEEE Fellow by Communications Society in 2007 for his "contributions to multi-user communications theory and cross-layer design of wireless networking standards".

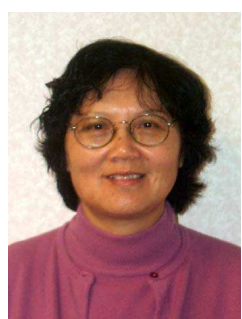

Jinyun Zhang received her B.Sc. degree in radio electronics from Tsinghua University, Beijing, China in 1970. Following her graduation, she was with Tsinghua University until 1985. She received her $\mathrm{Ph} . \mathrm{D}$. degree in electrical engineering from University of Ottawa, Canada in 1991. Dr. Zhang then joined Nortel Networks, where she held various management positions and engineering positions of increasing responsibility in the areas of digital signal processing, wireless communication and optical networks. Since 2001, Dr. Zhang has been the Manager of the Digital Communications \& Networking Group at Mitsubishi Electric Research Laboratories (MERL), Cambridge, MA, USA. Currently, she is leading various new broadband wireless communications and networking research projects that include UWB, ZigBee ad hoc networking, MIMO, broadband multimedia home networking, wireless sensor networks, high speed WLAN, cooperative communications, WiMAX and next generation mobile communications systems.

Dr. Zhang has authored and co-authored more than 130 publications, invented and co-invented more than 100 patents and patent applications, and made numerous contributions to international wireless communications standards. Dr. Zhang is a Fellow of the IEEE and a member of the IEEE AP, BT, COMM, IT, ITS, LEO, SP, and VT Societies. She serves as an Associate Editor of IEEE Transactions on Broadcasting, an AdCom member of IEEE Broadcasting Technology Society, and has served as a Technical Program Committee member for various IEEE conferences. 